\title{
Performance différenciée du coton Bt en début de diffusion: cas du Burkina Faso
}

\author{
Gaspard Vognan ${ }^{1}$ et Michel Fok ${ }^{2,3, *}$ \\ ${ }^{1}$ Programme coton, INERA, Bobo-Dioulaso, Burkina Faso \\ 2 CIRAD, UPR AIDA, F-34398 Montpellier, France \\ 3 AIDA, CIRAD, Univ Montpellier, Montpellier, France
}

\begin{abstract}
Résumé - L'avantage économique des cultures transgéniques pour les producteurs des pays en développement reste un sujet de préoccupation et de controverse. Seules quelques études ont pris en compte les différences entre producteurs pour comprendre les performances variables des cultures transgéniques, notamment dans le cas du coton Bt incorporant des gènes de la bactérie Bacillus thuringiensis pour la résistance à certains ravageurs du coton. Notre étude d'évaluation concerne le Burkina Faso où la diffusion à grande échelle du coton Bt a commencé en 2009 et a été suspendue en 2016. Elle aborde plus particulièrement les pratiques des producteurs, dans des exploitations différenciées selon le niveau d'équipement en culture attelée, la première année d'adoption à grande échelle du coton Bt et de diffusion de recommandations adaptées de traitement insecticide. Nous avons trouvé que le coton Bt augmentait les rendements, mais dans une moindre mesure que prévu; de plus, une augmentation de la rentabilité n'a été observée que dans les exploitations bien équipées en culture attelée (les plus aisées), mais pas pour les autres, plus démunies et représentant $58 \%$ des producteurs. Cela résulte des stratégies des producteurs démunis consistant à réduire les dépenses monétaires pour les engrais minéraux et les insecticides, stratégies exacerbées par le prix élevé des semences de coton Bt. Le cas étudié indique que les premiers impacts d'une nouvelle technologie dépendent de son prix et de la prise en compte, ou pas, du comportement des producteurs les plus démunis.
\end{abstract}

Mots clés : biotechnologies / pratiques culturales / rentabilité / différenciation des exploitations / OGM

Abstract - Differentiated early performance of Bt cotton: Case in Burkina Faso. The economic benefit of transgenic crops for producers in developing countries remains a matter of concern and controversy. Only a few studies have taken into account differences between producers to understand the variable effects of transgenic crops, particularly in the case of Bt cotton incorporating Bacillus thuringiensis genes for resistance to certain cotton pests. Our assessment study deals with Burkina Faso where the large-scale release of Bt cotton occurred in 2009, and was then suspended in 2016. It addresses the cultivation practices of producers, in farms differentiated by their level of ox-drawn equipment, on the first year of Bt cotton large adoption and dissemination of recommendations for an adapted insecticide protection. We found that $\mathrm{Bt}$ cotton increased yields, but to a lesser extent than expected; moreover, an increase in profitability was only observed in farms with a good ox-drawn equipment (the healthiest ones), but not for the others representing $58 \%$ of all farms. This is the result of worst-off producers' strategies, consisting in reducing cash expenses in fertilizers and insecticide, which were further exacerbated by the high price of Bt cottonseeds. The case study points out that the early impacts of a new technology depend on its price and on taking into account, or not, the behaviour of the poorest producers.

Keywords: biotechnologies / cultivation practices / profitability / farm differentiation / GMO

\footnotetext{
*Auteur de correspondance : michel.fok@cirad.fr
} 


\section{Introduction}

L'avantage économique des cultures transgéniques pour les utilisateurs des pays en développement est devenu un sujet de préoccupation et de controverse dès le début de leur mise sur le marché; cela concerne en particulier le coton Bt, commercialisé depuis 1998 pour contrôler certains ravageurs par incorporation de gènes de Bacillus thuringiensis. Si de nombreux observateurs ont estimé que l'adoption des biotechnologies en agriculture contribuerait à réduire la pauvreté (par exemple, pour les institutions internationales: Paarlberg, 2000 ; FAO, 2004 ; Persley, 2006), la controverse a été vive en Inde avant même la commercialisation officielle (Bharathan, 2000).

Les premières études sur le coton transgénique ont clamé sans nuance les impacts positifs pour les pauvres en Chine (Pray et al., 2001), en Afrique du Sud (Morse et al., 2004) ou en Inde (Barwale et al., 2004). Des opinions plus équilibrées sont apparues par la suite (Hofs et al., 2006; Gouse et al., 2005 ; Qaim et al., 2006; Pemsl et al., 2004); il existe en effet des contraintes socio-économiques spécifiques dans les pays en développement (Stone, 2004). L'optimisme sur les impacts positifs pour les pauvres persiste néanmoins (Paarlberg, 2008), ce qui a conduit Glover (2010) à dénoncer une construction narrative minimisant les aspects négatifs du coton $\mathrm{Bt}$.

Malgré le grand nombre d'études visant à évaluer les impacts du coton Bt dans les pays en développement, seules quelques-unes ont abordé l'influence des différences entre agriculteurs, et encore ont-elles surtout comparé les grandes exploitations entrepreneuriales et les petites exploitations familiales, là où elles coexistent. En Afrique du Sud, les auteurs ont conclu que les petits exploitants agricoles tiraient les mêmes avantages que les agriculteurs commerciaux (Morse et al., 2004), alors qu'en Argentine ce n'était pas le cas (Qaim et de Janvry, 2005).

Il existe encore moins d'études évaluant l'influence du type d'exploitation agricole familiale sur les avantages du coton Bt. En Inde, Qaim et al. (2006) ont souligné que «la technologie Bt pourrait ne pas convenir à tous les agriculteurs », en se fondant sur des facteurs techniques sans se pencher sur l'influence des facteurs structurels des exploitations agricoles. Morse et al. (2007) ont évoqué «l'effet paysan » dans le degré d'intensification de la culture du coton (irrigation et utilisation d'intrants) pour distinguer les adoptants et les non-adoptants du coton Bt, mais sans aborder les différences de performance observées. Au Burkina Faso, Renaudin et al. (2012) n'ont pas trouvé que le coton Bt induisait de gain de rendement, mais que ce rendement était positivement influencé par le niveau d'équipement en culture attelée. L'absence de gain de rendement avec le coton $\mathrm{Bt}$ ne permet pas de comprendre la position de certains paysans réclamant le retour du coton $\mathrm{Bt}$ dans un contexte de baisse de rendement (Gakpo, 2018), après sa suspension depuis 2016 pour des raisons financières liées au défaut de qualité de la fibre à l'exportation (Fok, 2016). L'effet positif du niveau d'équipement sur le rendement est conforme à l'observation de Berti et al. (2007), même si l'absence de relation est le plus souvent relevée dans les pays de l'Afrique francophone (Coulibaly et al., 1993 ; Fok, 1998, 2007).

Notre étude est une contribution pour compenser le manque de recherche sur l'influence des différences entre les producteurs de petite agriculture familiale dans l'évaluation du coton Bt. Elle se focalise sur la première année de diffusion pour cerner le degré d'application des recommandations dans les traitements insecticides et qui peut impacter la performance obtenue. Notre objectif est d'évaluer l'utilisation du coton Bt, en tenant compte de la différenciation des exploitations selon le niveau d'équipement en culture attelée. Notre étude permet de relier les pratiques culturales des producteurs en début de diffusion de ce coton, lors de la campagne 2009-2010, et de révéler des stratégies différenciées dans l'utilisation d'une technologie nouvelle exigeant dépense monétaire. L'étude apporte ainsi des enseignements utiles pour éclairer le débat actuel sur une reprise éventuelle de l'utilisation du coton-Bt dans le pays étudié.

\section{Matériels et méthodes}

\section{1 Étude dans une filière privatisée mais administrée}

L'étude est réalisée au Burkina Faso, dans une filière privatisée depuis 2005 avec trois sociétés cotonnières dont la Sofitex (Société burkinabè des fibres textiles) représentant environ $80 \%$ de la production du pays.

Pour notre étude, la caractéristique administrative du secteur du coton a facilité la collecte des coûts de production à dépense monétaire au niveau des agriculteurs. Le prix d'achat du coton-graine est en effet annoncé à l'avance et s'applique à tous les producteurs; les prix des engrais minéraux et des pesticides fournis à crédit sont déterminés avant le début de campagne et appliqués à tous les producteurs; le coût de ces intrants est déduit de la valeur des quantités de coton-graine fournies à la commercialisation.

\subsection{Source des données et typologie des exploitations}

Notre étude s'est appuyée sur les données de la campagne 2009-2010 issues du dispositif d'enquête permanente dans la zone cotonnière de la Sofitex. C'était la première année de diffusion à grande échelle du coton $\mathrm{Bt}$, cependant sans couverture totale des surfaces. La culture du coton conventionnel a été maintenue sur près de $60 \%$ des surfaces totales, avec la recommandation d'un programme insecticide à six traitements. Les données du dispositif d'enquête permanente pour les deux autres sociétés cotonnières n'ont pas été prises en compte parce que ces sociétés n'y ont cultivé que du coton Bt. Pour la culture du coton $\mathrm{Bt}$, devant permettre de contenir les attaques des chenilles habituellement contrôlées par les quatre premiers traitements insecticides, il a été recommandé de ne maintenir que les deux derniers traitements, visant les attaques des ravageurs piqueurs-suceurs; cette dernière recommandation a été cependant diffusée de manière tardive, voire confuse (Renaudin et al., 2012), ce qui a pu nuire à son application.

Les données ont concerné 134 exploitations réparties entre huit villages où le coton $\mathrm{Bt}$ et le coton conventionnel ont été cultivés en 2009. Les huit villages correspondaient à deux zones pluviométriques pour lesquelles deux variétés distinctes ont été vulgarisées.

Pour la typologie des exploitations agricoles, nous avons distingué trois types selon le niveau d'équipement en culture attelée, de manière assez similaire à Renaudin et al. (2012): exploitations à équipement multiple (au moins quatre bœufs et 
deux charrues, soit au moins deux attelages); exploitations à équipement simple ( 2 ou 3 bœufs et une charrue, soit un attelage); exploitations à équipement incomplet (au plus 1 bœuf, avec ou sans charrue). Les exploitations à équipement incomplet peuvent cependant pratiquer aussi la culture attelée en empruntant ou en louant des attelages, mais de manière non optimale. Les exploitations à équipement multiple sont considérées comme les plus aisées dans la zone cotonnière du Burkina Faso, alors que celles à équipement incomplet sont les plus pauvres (Renaudin, 2010).

$\mathrm{Au}$ sein de chaque exploitation, plusieurs parcelles de coton, de taille variable, peuvent être semées et conduites de manière différente, notamment pour le type de coton utilisé $(\mathrm{Bt}$ ou non $\mathrm{Bt}$ ). Leur suivi tout au long du cycle, avec recueil régulier des données, a été réalisé pour 185 parcelles de coton (cultivées par 134 exploitations).

\subsection{Variétés cultivées et semences}

Au Burkina Faso, jusqu'à l'adoption du coton Bt et même quelques années après, les variétés distribuées aux producteurs l'ont été en tenant compte de deux zones pluviométriques. La variété STAM 59A était dédiée aux zones moins pluvieuses, notamment la partie nord de la zone Sofitex, mais elle était moins répandue que l'autre variété, FK37. Afin de pouvoir utiliser des variétés adaptées aux conditions locales, le Burkina Faso avait fait transformer en coton Bt ces deux variétés; il disposait ainsi de deux couples de variétés isogéniques, chaque couple étant constitué de la variété Bt et non-Bt.

L'originalité de notre étude est qu'elle compare deux couples de variétés isogéniques de coton $\mathrm{Bt}$ et de coton non-Bt (expression plus précise que «conventionnel», puisque les variétés ne se distinguent que par la présence ou l'absence des gènes $\mathrm{Bt}$ ).

Le dispositif de l'étude n'était cependant pas parfaitement équilibré, car tous les producteurs n'ont pas cultivé les deux types de coton (Bt et non-Bt) l'année étudiée; cela a été seulement le cas des plus grandes exploitations, les autres ayant cultivé seulement l'un ou l'autre type.

La diffusion du coton $\mathrm{Bt}$ a entraîné une augmentation spectaculaire du prix des semences par rapport au coton nonBt. Les semences de coton Bt étaient distribuées seulement sous forme délintée, à 2250 FCFA/kg (soit $27000 \mathrm{FCFA} / \mathrm{ha}$ pour $12 \mathrm{~kg} / \mathrm{ha}, 1 \mathrm{USD}=470 \mathrm{FCFA}$ ), contre $70 \mathrm{FCFA} / \mathrm{kg}$ pour le coton non-Bt (distribué aussi, plus fréquemment, sous forme vêtue, à $30 \mathrm{FCFA} / \mathrm{kg}$ pour $20 \mathrm{~kg} / \mathrm{ha}$ ).

\subsection{Entités comparées et indicateurs de comparaison}

Pour évaluer la performance du coton $\mathrm{Bt}$, ont été comparées les 185 parcelles de coton $\mathrm{Bt}$ et de coton non-Bt, relevant d'exploitations ayant cultivé soit l'un des deux types de coton, soit les deux. Seules 22 exploitations (sur 134) ont cultivé les deux types de coton; 62 et 50 exploitations ont cultivé respectivement du coton Bt seul ou du non-Bt seul. Dans les comparaisons réalisées, les 22 exploitations avec les deux types de coton ont été dédoublées avec les parcelles de coton $\mathrm{Bt}$ et non-Bt affectées aux deux groupes d'exploitations selon le type de coton. On a ainsi un groupe de $84(62+22)$ exploitations à coton $\mathrm{Bt}$ et un groupe de $72(50+22)$ exploitations à coton non-Bt.
La performance du coton Bt est évaluée par comparaison avec les parcelles de coton non-Bt, selon le rendement, les coûts des intrants et la marge brute ; cette dernière est estimée par approximation à partir de la valeur de la production moins le coût total des semences et des produits chimiques (engrais, insecticides et herbicides), soit la marge après remboursement des intrants (MARI). Nous avons aussi calculé le coût du contrôle des ravageurs, indicateur issu de la somme du coût des semences et des insecticides. Cet indicateur est habituellement utilisé dans les études d'évaluation du coton Bt (Smale et al., 2010). La rétribution de la main-d'œuvre non familiale n'a pas été prise en compte. L'incidence éventuelle d'un coût plus élevé en main-d'œuvre temporaire recrutée pour la récolte d'une production plus élevée, comme cela était attendu pour le coton $\mathrm{Bt}$, est donc occultée. Il en découle que le profit relatif du coton $\mathrm{Bt}$, si profit il y a, serait plutôt surestimé.

Comme nous avons pris en compte les coûts au-delà de ceux liés à la lutte antiparasitaire, notre approche pour évaluer le coton Bt était plus complète que la méthode du budget partiel adoptée dans de nombreuses études antérieures (Smale et al., 2010). L'approche du budget partiel suppose que les adoptants d'une technologie ne modifient que leurs pratiques directement liées à la technologie, c'est-à-dire, dans notre cas, la lutte antiparasitaire. Notre approche permet de voir si les producteurs modifient aussi certaines pratiques culturales non directement liées à la technologie du coton $\mathrm{Bt}$, comme cela a été observé en Inde dans les premières années de culture de ce type de coton (Morse et al., 2007).

Les indicateurs pour apprécier la performance du coton Bt, rendement et marge brute, dépendent potentiellement des types et des variétés de coton utilisés, mais aussi de facteurs structurels de l'exploitation (superficie totale cultivée, taille de la famille, équipement) et des pratiques culturales (superficie cotonnière, période des semis, fumure organique, fertilisation minérale, nombre de pulvérisations d'insecticide et coût du contrôle des ravageurs). L'incidence des divers facteurs pris dans leur ensemble est vérifiée par régression multiple, après s'être assuré de ne retenir que ceux qui sont indépendants. Notre approche est ainsi plus complète que celle de Renaudin et al. (2012) qui n'ont considéré que deux variables explicatives (niveau d'équipement et type de coton).

\section{Résultats}

\subsection{Caractéristiques des exploitations}

Les trois niveaux d'équipement étaient représentés dans les 134 exploitations étudiées (Tab. 1), même si les exploitations à équipement incomplet étaient moins fréquentes. Le niveau d'équipement et les autres caractéristiques des exploitations sont corrélés, ce qui réduit le nombre de variables explicatives à considérer dans les régressions multiples : un meilleur niveau d'équipement correspond à de plus grandes exploitations, familles et soles cotonnières. Les différences dans l'âge du chef d'exploitation et dans la part du coton dans l'assolement étaient perceptibles mais non significatives.

La fréquence des exploitations ayant cultivé les deux types de coton (Tab. $2,4^{\mathrm{e}}$ colonne) était trop faible ( 22 exploitations, soit $16 \%$ ) pour nous permettre de les traiter séparément. Les deux groupes, Bt et non-Bt, avaient des effectifs proches (84 et 72), mais les exploitations du groupe Bt étaient de plus grande 
Tableau 1. Caractéristiques des exploitations selon leur niveau d'équipement.

Table 1. Farm features according to their equipment level.

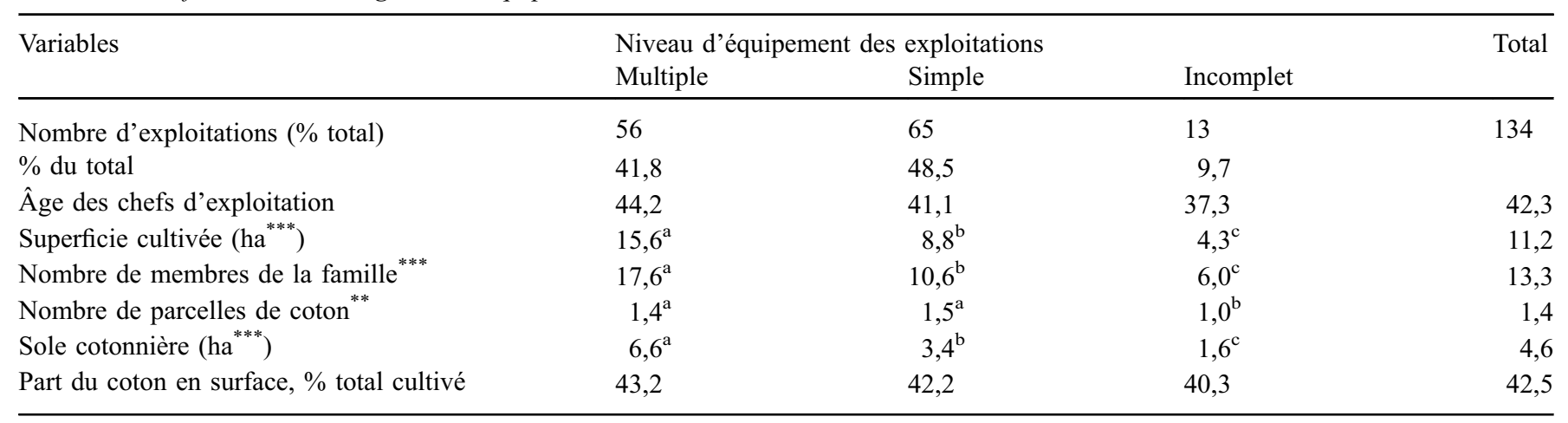

${ }^{*}$ significatif à $5 \% ;^{* *}$ significatif à $1 \% ;{ }^{* * *}$ significatif à moins de $0,1 \%$. Les lettres distinguent les moyennes comparées deux à deux.

Tableau 2. Caractéristiques des exploitations et pratiques culturales selon le type de coton et les groupes d'exploitation.

Table 2. Farm features and cultivation practices according to the type of cotton grown and farm groups.

\begin{tabular}{|c|c|c|c|c|c|c|}
\hline Variables & \multicolumn{3}{|c|}{ Exploitations de l'échantillon } & \multicolumn{3}{|c|}{ Exploitations selon le type de coton cultivé } \\
\hline Superficie cultivée (ha) & 12,1 & $6,6^{* * *}$ & 12,0 & 12,1 & $8,9^{* * *}$ & \\
\hline Nombre de membres de la famille & 14,5 & $11,0^{* * *}$ & 15,2 & 14,6 & $12,3^{* * *}$ & \\
\hline Surface coton/total $(\%)$ & 43,8 & 35,0 & 43,9 & 43,9 & 38,8 & \\
\hline \multicolumn{7}{|l|}{ Proportion $(\%)$} \\
\hline Semis à la période recommandée & & & & 79,2 & 74,7 & 77,6 \\
\hline Apport de fumure organique & & & & 16,8 & 27,8 & 21,8 \\
\hline Apport d'engrais complexe & & & & 100,0 & $86,3^{* *}$ & 93,6 \\
\hline Nombre de traitements insecticides & & & & 1,3 & $5,1^{* * *}$ & 3,3 \\
\hline Dose d'engrais complexe (kg/ha) & & & & 126 & $111^{* * *}$ & 119 \\
\hline Dosée d'urée (kg/ha) & & & & 46 & 44 & 45 \\
\hline
\end{tabular}

Les nombres d'exploitations selon le type de coton cultivé sont obtenus en affectant les 22 exploitations ayant cultivé les deux types de coton dans chacun des deux groupes d'exploitation. Comparaisons faites entre les exploitations selon les deux types de coton au sein de chaque groupe. ${ }^{*},{ }^{* *}$ et ${ }^{* * *}$ pour signification statistique respectivement à $5 \%, 1 \%$ et $0,1 \%$.

taille, en démographie, en nombre de parcelles de coton, en surface cultivée et en sole cotonnière (Tab. 2).

\subsection{Pratiques culturales}

Il est communément constaté que certaines pratiques peuvent avoir un impact sur la performance de la culture du coton, notamment la période des semis, l'utilisation de fumure organique, la fertilisation minérale et la lutte antiparasitaire (Fok, 2007). Dans notre enquête, des différences ont été observées dans ces pratiques entre les deux groupes, $\mathrm{Bt}$ et non- $\mathrm{Bt}$, quoique de manière non systématique (Tab. 2). Les deux types d'exploitation ne diffèrent pas pour la période de semis, ni pour la fréquence (faible, 17 à $28 \%$ ) d'apport de fumure organique.

Par contre, les exploitations à coton non-Bt se distinguent par divers indicateurs d'intensification moindre lorsque celleci repose sur des apports d'intrants à dépense monétaire. Cela est manifeste pour l'utilisation des engrais. Ces exploitations ont apporté moins fréquemment de l'engrais complexe et de l'urée. La dose d'engrais complexe a été plus faible; la dose d'urée a aussi été moindre mais pas de manière significative.

Alors que quasiment toutes les exploitations à coton $\mathrm{Bt}$ ont apporté de l'urée, ce n'est pas le cas pour un quart des exploitations à coton non-Bt. L'absence d'apport d'urée est 
Tableau 3. Absence d'apport d'urée et de traitement insecticide avec coton Bt, selon le type d'exploitation.

Table 3. No use of urea and insecticide spray according to farm types.

\begin{tabular}{|c|c|c|c|c|c|c|}
\hline \multirow[t]{2}{*}{ Exploitations } & \multirow[t]{2}{*}{ Total } & \multicolumn{3}{|c|}{ Niveau d'équipement } & \multicolumn{2}{|c|}{ Exploitations dans la zone } \\
\hline & & Multiple & Simple & Incomplet & Sud & Nord \\
\hline Nombre total & 134 & 56 & 65 & 13 & 102 & 54 \\
\hline Fréquence $(\%)$ & 17,2 & 14,3 & 15,4 & 38,5 & 4,9 & 33,3 \\
\hline Valeur de $p$ & & \multicolumn{2}{|c|}{0,099} & & \multicolumn{2}{|c|}{$<0,0001$} \\
\hline$\%$ sans traitement insec. & 37,4 & 36,7 & 31,8 & 83,3 & & \\
\hline Valeur de $p$ & \multicolumn{4}{|c|}{0,050} & & \\
\hline
\end{tabular}

Tableau 4. Coût de production et performance selon le niveau d'équipement et le type de coton.

Table 4. Production cost and performance according to equipment level and type of cotton.

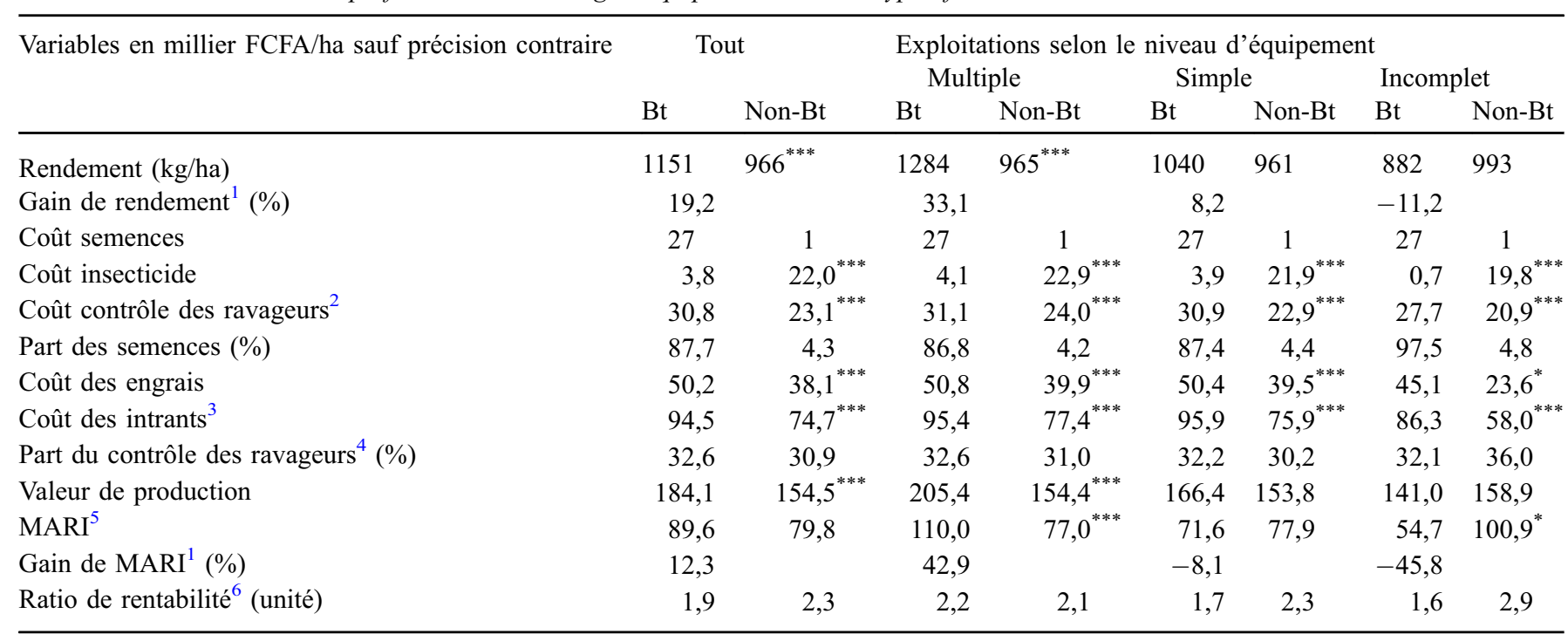

${ }^{* *},{ }^{* *}$ et ${ }^{* * *}$ pour signification à $5 \%, 1 \%$ et moins que $0,1 \%$.

${ }^{1}$ Différence procurée par le coton $\mathrm{Bt}$ et rapportée au coton non-Bt.

${ }^{2}$ Total des coûts des semences et des insecticides.

${ }^{3}$ Coût incluant celui des herbicides, non présenté ici.

${ }^{4}$ Rapport du coût du contrôle des ravageurs sur le coût total des intrants.

${ }^{5}$ Marge après remboursement des intrants.

${ }^{6}$ Rapport de la valeur de la production sur le coût des intrants.

observée pour les trois niveaux d'équipement en culture attelée, mais est plus fréquente quand l'équipement est incomplet (Tab. 3). Au regard des deux zones géographiques, dans lesquelles deux variétés distinctes ont été cultivées, l'absence d'apport d'urée a été observée bien plus fréquemment au nord, moins pluvieux, dans une exploitation sur trois.

L'appréciation sur les pratiques de traitement insecticide est plus délicate car la culture des deux types de coton était accompagnée de recommandations distinctes. Il est donc normal que le nombre moyen de traitements soit supérieur pour le coton non-Bt $(5,1$ contre 1,3$)$. Ces nombres sont inférieurs aux recommandations respectives de 6 et 2 . Les exploitations respectant exactement les nombres recommandés étaient minoritaires, mais sans différence significative entre $\mathrm{Bt}$ et non-Bt. Avec le coton non-Bt, aucun cas d'absence de traitement insecticide n'a été observé, alors que cela a été le cas pour plus du tiers des parcelles de coton Bt; c'est davantage le cas des exploitations à équipement incomplet (cinq des six exploitations concernées, Tab. 3).

\subsection{Rendement, coût de production et rentabilité}

Globalement, le coton $\mathrm{Bt}$ a induit un gain de rendement de $19 \%$ (Tab. 4), mais ce gain varie selon le niveau d'équipement 
Tableau 5. Effet de la zone-variété sur la performance.

Table 5. Effect of zone-variety on performance.

\begin{tabular}{|c|c|c|c|c|c|}
\hline & \multirow[t]{2}{*}{ Types d'exploitation } & \multicolumn{2}{|c|}{ SUD-FK 37} & \multicolumn{2}{|c|}{ NORD-STAM 59A } \\
\hline & & $\mathrm{Bt}$ & Non-Bt & $\mathrm{Bt}$ & Non-Bt \\
\hline \multirow{2}{*}{ Nombre d'exploitations } & Total & 60 & 42 & 24 & 30 \\
\hline & Équipement simple/incomplet & 32 & 24 & 10 & 23 \\
\hline \multirow[b]{2}{*}{ Rendement (kg/ha) } & Total & 1035 & $934^{*}$ & 1441 & $1011^{* * *}$ \\
\hline & Équipement multiple & 1173 & $948^{* * *}$ & 1506 & $1009^{* * *}$ \\
\hline \multirow{3}{*}{ Coût des intrants (milliers FCFA) } & Total & 90,8 & $85,3^{* *}$ & 101,4 & $59,8^{* * *}$ \\
\hline & Équipement multiple & 92,2 & $84,3^{* *}$ & 101,9 & $59,7^{* *}$ \\
\hline & Équipement simple/incomplet & 89,7 & 86,2 & 100,7 & $59,8^{* * *}$ \\
\hline \multirow[b]{2}{*}{ MARI (milliers FCFA/ha) } & Total & 72,3 & 64,0 & 129,2 & $101,9^{*}$ \\
\hline & Équipement multiple & 95,4 & $67,4^{* *}$ & 139,2 & $101,7^{*}$ \\
\hline
\end{tabular}

Comparaison entre les deux types de coton à l'intérieur de chaque zone-variété : ${ }^{*},{ }^{* *},{ }^{* * *}$ : pour signification statistique à $5 \%, 1 \%$ et moins de $0,1 \%$.

des exploitations. Dans les exploitations à équipement multiple, plus aisées, le coton $\mathrm{Bt}$ a procuré le gain de rendement le plus élevé (différence de $33 \%$, significative avec valeur de $p<0,01 \%$ ) alors qu'aucun gain significatif n'a été observé dans les autres exploitations. Dans les exploitations à équipement incomplet, le rendement était même arithmétiquement plus élevé lorsque le coton non-Bt était cultivé.

Le rendement plus élevé du coton Bt a été associé à des dépenses plus élevées pour la lutte contre les ravageurs, quel que soit le niveau d'équipement. Dans les parcelles de coton $\mathrm{Bt}$, le coût du contrôle des ravageurs s'est élevé à $33 \%$ du coût total des intrants pour l'ensemble des trois niveaux d'équipement, légèrement plus élevé que dans les parcelles de coton non-Bt $(31 \%) \mathrm{du}$ fait de l'incidence des semences Bt. Les écarts observés entre les parcelles Bt et non-Bt concernent les exploitations à équipement simple ou multiple, mais pas les exploitations à équipement incomplet, car le coût en insecticides y était très faible sur les parcelles de coton Bt. Les semences comptent pour $88 \%$ du coût total engagé contre les ravageurs dans les parcelles de coton $\mathrm{Bt}$, alors qu'elles ne représentent que $4,3 \%$ dans les parcelles de coton non-Bt. Cette part prépondérante des semences dans le coût du contrôle des ravageurs était encore plus marquée dans les exploitations à équipement incomplet.

Le coût des engrais était aussi plus élevé dans les parcelles de coton $\mathrm{Bt}, 32 \%$ de plus que dans les parcelles de coton nonBt pour l'ensemble des exploitations. L'écart était cependant bien plus grand dans le cas des exploitations à équipement incomplet $(+91 \%)$ car le coût des engrais y était beaucoup plus faible dans les parcelles de coton non-Bt.

Globalement, les coûts des intrants pour les parcelles de coton $\mathrm{Bt}$ étaient supérieurs à ceux des parcelles de coton nonBt. La différence des coûts était de $27 \%$ pour l'ensemble des trois niveaux d'équipement, mais elle était nettement plus forte dans le cas des exploitations à équipement incomplet (49\%).

Pour l'ensemble des trois types d'exploitations, le coton $\mathrm{Bt}$ n'a pas induit de gain de marge après remboursement des intrants pris à crédit (MARI). Seules les exploitations à équipement multiple, les plus aisées, ont enregistré un gain important $(+43 \%)$ grâce au coton Bt. À l'opposé, dans les exploitations à équipement incomplet, l'utilisation du coton $\mathrm{Bt}$ a induit une perte de marge significative, de $46 \%$. Pour les exploitations à équipement simple, une perte de marge a également été observée, mais non significative.

Selon le ratio de rentabilité, défini ici comme étant le rapport entre la valeur de la production et le coût des intrants et considéré comme un indicateur de risque financier, l'adoption du coton Bt était associée à un risque financier plus élevé. Moins les exploitations étaient équipées, plus le risque était élevé. En référence à la valeur de 3,0 généralement considérée pour juger de l'acceptabilité de l'intensification agricole dans les pays en développement et qui a guidé les actions en Afrique francophone (CFDT, 1995 ; Fichet, 1996), la culture du coton paraissait déjà risquée avec le coton non-Bt (ratio de 2,3) mais elle le devenait davantage avec le coton $\mathrm{Bt}(1,9)$. À noter que le ratio de rentabilité retenu est aussi un indicateur de la productivité de tous les intrants. À cet égard, le coton Bt n'a pas entraîné d'augmentation de cette productivité.

\subsection{Effet des zones et des variétés}

Comme les deux variétés ont été distribuées à deux zones pluviométriques spécifiques, les effets entre zones et variétés ne pouvaient normalement pas être séparés dans notre étude. Cependant, comme la variété STAM-59A a été utilisée dans la zone Nord de la Sofitex avec une écologie moins favorable, où de surcroît les producteurs étaient plus précautionneux dans l'utilisation des intrants (cf. supra à propos de l'urée), si une plus grande performance a été tout de même observée, on peut présumer qu'elle procédait surtout de la variété.

En effet, la performance de la variété STAM-59A dans la zone Nord a été meilleure que celle de la variété FK-37 dans la zone Sud, en termes de rendement et de MARI, et ce pour les deux types de coton, Bt ou non-Bt (Tab. 5). Le gain de rendement apporté par le coton Bt était aussi plus grand avec la 
Tableau 6. Facteurs explicatifs du rendement et de la marge (MARI). Table 6. Explaining factors of yield and margin.

\begin{tabular}{lcc}
\hline Variables explicatives & \multicolumn{2}{c}{ Variables à expliquer } \\
\cline { 2 - 3 } & Rendement & MARI \\
\hline Niveau d'équipement ${ }^{1}$ & $-0,279^{* * *}$ & $-0,297^{* * *}$ \\
Simple & $-0,167^{* *}$ & $-0,178^{* *}$ \\
Incomplet & 0,0296 & 0,031 \\
Apport de fumure organique & 0,061 & 0,065 \\
Semis à période recommandée & $0,739^{* *}$ & $0,785^{* *}$ \\
Coton Bt & $0,447^{* * *}$ & $0,475^{* * *}$ \\
Variété Stam-59A & $0,522^{* *}$ & $0,555^{* *}$ \\
Nombre de traitements insecticides & $-0,070$ & $-0,276^{* *}$ \\
Coût du contrôle des ravageurs & $0,317^{* * *}$ & $-0,021$ \\
Coût des engrais & 0,498 & 0,434 \\
$\mathrm{R}^{2}$ & & \\
\hline
\end{tabular}

${ }^{*},{ }^{* *},{ }^{* * *}$ : pour signification statistique à $5 \%, 1 \%$ et moins de $0,1 \%$. ${ }^{1}$ Relativement au niveau d'équipement multiple.

variété STAM-59A, dans la zone Nord. Ce gain était de $43 \%$ pour les trois niveaux d'équipement pris ensemble, avec un écart plus grand pour les exploitations à équipement multiple (49\% contre $34 \%$ pour les autres).

La supériorité en termes de MARI avec la variété STAM59A dans la zone Nord a été de 79 et $59 \%$, respectivement pour les parcelles en coton Bt et en coton non-Bt, tous niveaux d'équipement confondus. Par contre, l'ampleur du gain en MARI procuré par le type Bt, même avec la variété STAM59A dans la zone Nord, était plus faible et variable selon le niveau d'équipement des exploitations. Ce gain était de $27 \%$ pour l'ensemble des exploitations, avec un gain de $37 \%$ dans les exploitations à équipement multiple et un gain non significatif dans les autres.

\subsection{Analyse multivariée}

L'analyse multivariée confirme les éléments précédents de manière hautement significative (Tab. 6). Le rendement et l'indicateur de rentabilité MARI sont positivement impactés par le niveau d'équipement, par le type $\mathrm{Bt}$, et par la variété STAM-59A. Le nombre de traitements insecticides a un impact positif sur le rendement et la marge MARI, l'utilisation plus forte d'engrais favorise le rendement mais est sans effet sur la MARI, alors que le coût du contrôle des ravageurs n'a pas d'effet sur le rendement et un effet négatif sur la MARI. L'apport de fumure organique et le semis à la période recommandée n'ont pas d'incidence.

\section{Discussions}

\subsection{Gain avec le coton $\mathrm{Bt}$, mais faible et variable selon les exploitations}

Avec un niveau de rendement du coton non-Bt conforme à l'évolution observée (Berti et al., 2007), mais supérieur au rendement moyen pour l'ensemble du pays pour la campagne
2009-2010 - $867 \mathrm{~kg} / \mathrm{ha}$ selon Fok (2016) et les statistiques de l'ICAC (avec conversion en rendements de coton-graine) notre étude montre que le coton Bt a apporté un gain de $19 \%$ lorsque tous les producteurs sont considérés dans leur ensemble. Ce résultat est contraire à celui de Renaudin et al. (2012), qui n'avaient pas pris en compte que deux variétés à performance bien distincte ont été cultivées. Comme leur niveau de rendement du coton non-Bt était anormalement élevé (rendement de $1071 \mathrm{~kg} / \mathrm{ha}$ contre 966 dans notre étude, pour la même campagne), cela peut venir du fait que ces auteurs ont comparé du coton non-Bt correspondant essentiellement à la variété STAM-59A avec du coton Bt relevant essentiellement de la variété FK-37.

Le gain observé dans notre étude est très variable selon les producteurs: meilleur chez ceux disposant d'équipement multiple et qui sont plus aisés (33\%) et faible, voire négatif chez les autres. La typologie des exploitations selon trois niveaux d'équipement a donc bien discriminé les exploitations selon leur structure (Tab. 1), mais aussi selon leurs pratiques culturales et leurs performances dans la culture du coton $\mathrm{Bt}$ (Tab. 2 et 4$)$.

\subsection{Impact du coton Bt probablement surestimé par l'échantillon de l'étude}

Le recours aux données de l'enquête permanente a certes été approprié pour intégrer la diversité des producteurs utilisant du coton $\mathrm{Bt}$, mais l'échantillon était probablement un peu biaisé, ce qui concourt à surestimer l'impact du coton Bt. L'enquête permanente a été mise en place pour suivre la rentabilité de la production du coton dans les exploitations selon leur niveau d'équipement en culture attelée, mais la répartition des exploitations selon leur équipement a probablement été impactée par leur participation à l'enquête permanente. En effet, les participants à l'enquête permanente ont bénéficié d'une interaction plus fréquente avec les techniciens et les scientifiques impliqués que les autres exploitants, ce qui a pu être favorable à leurs résultats (rendement moyen supérieur au rendement sur l'ensemble du Burkina Faso) et donc à leur capacité à s'équiper. Ainsi peut-on comprendre la faible proportion des exploitations à équipement incomplet, $10 \%$ ici contre $32,0 \%$ selon Renaudin (2010). L'échantillon de notre étude contenait donc une proportion plus importante d'exploitations équipées, et particulièrement de celles à équipement multiple, que dans l'ensemble des producteurs de coton. Un tel biais surestime l'impact global du coton $\mathrm{Bt}$, car les exploitations mieux équipées tirent davantage de profit du coton Bt. Le gain de rendement de 19\% trouvé dans notre étude est donc probablement supérieur à la réalité. Le gain de rendement a été par ailleurs aussi surestimé dans notre étude par la part des surfaces semées avec la variété STAM-59A, utilisée par 35\% de nos exploitations contre 20-25\% dans la réalité, alors que cette variété s'est révélée plus performante.

\subsection{Effet variétal}

Notre étude respecte la rigueur souhaitée de comparer des variétés isogéniques dans l'évaluation du coton $\mathrm{Bt}$ (Fok, 2006). 
Nous avons en effet comparé ici des variétés isogéniques, ne se distinguant que par la présence/absence des gènes $\mathrm{Bt}$, contrairement à la plupart des études d'évaluation du coton Bt, comme l'avait relevé Smale et al. (2010).

Mieux encore, nous avons pu comparer deux couples de variétés isogéniques et constater que la différence de performance entre coton $\mathrm{Bt}$ et coton non-Bt peut dépendre de la variété. La variété STAM-59A a été nettement plus productive que la variété FK-37, alors qu'elle a été bien moins semée à l'échelle de tout le territoire (20-25\% des surfaces contre $35 \%$ ici). De manière plus générale, cela montre que les résultats d'une évaluation du coton $\mathrm{Bt}$ peuvent dépendre du degré d'adaptation des variétés utilisées lors de l'évaluation. Les effets du coton Bt peuvent être minorés si des variétés exogènes peu adaptées sont utilisées, ce qui peut être le cas en début de leur utilisation dans divers pays.

\subsection{Avantage du coton Bt plus faible qu'escompté}

Globalement, le coton $\mathrm{Bt}$ a induit un gain de rendement significatif de 19\%; il est inférieur (en dépit d'une certaine surestimation par le biais de l'échantillon) aux attentes initiales, alors que le gain de MARI $(+12 \%)$ n'est pas statistiquement significatif. Les décideurs de la filière coton attendaient un gain moyen de $30 \%$ en rendement et une augmentation en conséquence des revenus des producteurs (Baillard, 2006), même si un gain de $20 \%$ avait été rapporté dans les expérimentations préalables au Burkina Faso (Vitale et al., 2008). L'étude de Cabanilla et al. (2005) faite au Mali, mais avec extrapolation à l'Afrique de l'Ouest, avait même considéré que le gain de rendement pouvait atteindre $45 \%$.

La faiblesse du gain moyen de rendement procuré par le coton Bt a été observée en dépit du fait que les producteurs les plus nantis ont eu tendance à lui apporter des engrais minéraux de manière plus systématique et à plus forte dose (Tab. 2). Cela se traduit par des coûts en engrais minéraux plus élevés dans les parcelles en coton $\mathrm{Bt}$ (Tab. 4), mais qui restent payants avec une MARI plus élevée obtenue sur coton Bt, même si le niveau de signification statistique du gain observé (par rapport au coton non-Bt) est plus faible que pour le rendement (Tab. 5).

La faiblesse des effets positifs en début de diffusion du coton Bt est opposée à ce qui a été observé dans d'autres pays à petite agriculture familiale comme en Inde ou en Chine. Les facteurs d'influence peuvent être une pression assez faible des ravageurs au Burkina Faso, en tout cas bien moindre que dans ces deux pays d'Asie où la résistance des ravageurs aux insecticides était devenue très forte et n'était pas gérée. Si tel était le cas, le niveau initial d'infestation des ravageurs cibles du coton Bt serait un critère fondamental de la rentabilité de son utilisation.

Un autre facteur peut être lié au degré insuffisant d'achèvement de la transformation biotechnologique des variétés locales de coton fournies par le Burkina Faso (Fok, 2016). Nous manquons cependant de données pour étayer cette hypothèse.

\subsection{Des effets différenciés selon les stratégies des exploitations}

La performance du coton Bt varie selon les exploitations et seules celles à équipement multiple ont gagné en rendement
$(+33 \%)$ et en rentabilité $(+43 \%)$ dans des proportions correspondant aux attentes. Pour les autres, qui représentaient $58 \%$ des exploitations de l'étude, l'adoption du coton Bt n'a pas été rentable et elle a augmenté le risque financier associé à la culture du coton. Le désavantage financier est particulièrement évident chez les exploitations à équipement incomplet, les plus démunies financièrement.

Les résultats de notre étude donnent des éléments pour expliquer la différenciation des effets du coton Bt par des différences de stratégies d'intensification dans l'utilisation des intrants à sorties monétaires, du moins lors de la première année d'utilisation. Pour l'utilisation des engrais, nous avons vu que les producteurs y recouraient plus systématiquement dans les parcelles de coton Bt et avec des doses plus élevées, mais que cela était réalisé à un degré moindre dans les exploitations à équipement incomplet (Tab. 5). Ces dernières exploitations sont notamment plus nombreuses à ne pas apporter d'urée quand elles sont localisées dans le nord de la zone d'étude, où la perception du risque climatique est plus forte (Tab. 3). Pour les insecticides, ces mêmes exploitations étaient plus nombreuses à ne pas réaliser de traitement sur les parcelles de coton Bt.

On voit donc que les producteurs les plus démunis ont cherché à économiser les intrants nécessitant des sorties monétaires, comme pour compenser le surcoût consenti pour utiliser les semences de coton Bt. À l'opposé, les exploitations à équipement multiple, aux ressources financières moins limitées, ont cherché à «mettre le paquet» pour faire s'exprimer au mieux le potentiel attendu du coton Bt. La stratégie de ces dernières paraît plutôt payante si l'on se réfère à la valeur de la MARI plus élevée que pour les autres exploitations, mais elle implique d'accepter un risque financier plus important.

\subsection{Stratégie d'économie exacerbée par le prix de la technologie Bt}

Le coût de la technologie était élevé et il a été répercuté intégralement sur les agriculteurs. Le coût des semences est ainsi passé de $1000 \mathrm{FCFA} /$ ha en coton non-Bt à $27000 \mathrm{FCFA} /$ ha en BT, soit $4,75 \mathrm{USD} / \mathrm{kg}$. C'est un prix proche de celui appliqué dans les pays développés, et sensiblement plus élevé que les 3,9 USD/kg observés en 2005 en Afrique du Sud (Hofs et al., 2006). Un tel niveau de prix a probablement renforcé la stratégie des producteurs démunis basée sur une réduction des coûts des engrais et des insecticides.

La rentabilité d'ensemble du coton Bt a été affaiblie par le niveau élevé du prix de la technologie Bt, plus élevé que les économies que celle-ci permet de réaliser en insecticide. L'utilisation du coton Bt était censée permettre d'économiser quatre pulvérisations d'insecticide, dont le coût est de $17368 \mathrm{CFA} / \mathrm{ha}$, bien inférieur au coût des semences de $27000 \mathrm{CFA} / \mathrm{ha}$. La différence découle de la fixation de ce dernier coût selon l'accord entre le Burkina Faso et le fournisseur de la technologie Bt (Monsanto) et dont les modalités ont été succinctement indiquées par Renaudin et al. (2012). Dans ces modalités, la valeur d'une partie du gain espéré de $30 \%$ en rendement au niveau des paysans est attribuée aux acteurs de la fourniture des semences Bt. Une telle attribution est une pratique discutable - quoique peu/pas 
discutée - car le gain de productivité ne dépend pas seulement des semences utilisées mais aussi des compétences culturales des producteurs et de leurs investissements dans les autres intrants.

\subsection{Impacts d'une technologie nouvelle et de son prix}

Dans le cas du Burkina Faso, l'intégration (dans la fixation du prix de cette technologie) d'une anticipation de gain de rendement par le coton $\mathrm{Bt}$ a eu comme résultat de ne pas faire se concrétiser cette anticipation. C'est la conséquence de la réaction, non anticipée, des producteurs les plus démunis face au prix des semences. En réagissant par une utilisation moindre que ce qui est recommandé des engrais et des insecticides, ils contribuent à ne pouvoir atteindre les gains de rendement escomptés.

Notre étude confirme qu'en matière d'impacts d'une technologie nouvelle, le facteur prix de la technologie est fondamental, comme Graff et al. (2006) l'avaient souligné. Pour que de nouvelles technologies puissent bénéficier aux pauvres, leur tarification doit être adaptée aux conditions de ces derniers, cela renvoie à une dimension politique mentionnée par Scoones (2006) et que Herring (2007) a observée dans la régulation du coton $\mathrm{Bt}$ en Inde en 2006, quand le gouvernement central a forcé avec succès les distributeurs de semences $\mathrm{Bt}$ à réduire de moitié les prix (Gruère et al., 2008).

\section{Conclusion}

Notre étude a concerné la campagne 2009-2010, lors de la première campagne de diffusion effective et à grande échelle du coton $\mathrm{Bt}$ au Burkina Faso. Nous avons trouvé que le coton Bt pouvait apporter un gain, mais plus faible qu'escompté et variant fortement selon les exploitations, en fonction de leur niveau de capital traduit par leur niveau d'équipement en culture attelée, au point qu'il est nul pour les exploitations moins bien équipées et même négatif pour les exploitations incomplètement équipées.

Le bénéfice financier à court terme du coton Bt n'a pas répondu aux attentes de l'ensemble des producteurs. Les différences observées dans l'utilisation de ce coton, au début de sa diffusion, procédaient de divergences de stratégies des exploitations. Les exploitations à équipement multiple - les mieux loties - ont obtenu le gain escompté parce qu'elles ont davantage investi dans les intrants et ont accepté des risques financiers plus élevés. À l'opposé, leur perception du risque financier a amené les exploitations à équipement incomplet, plus démunies, à économiser sur les engrais et insecticides pour compenser le surcoût payé pour les semences.

Dans des pays en développement, l'impact d'une technologie nouvelle, comme les cultures transgéniques, dépend notamment du prix pour y accéder et de l'adaptation que ce prix induit chez les producteurs, selon deux stratégies opposées bien mises en évidence ici. Au Burkina Faso, nos résultats montrent que le prix de la technologie $\mathrm{Bt}$ a été trop élevé pour les producteurs; son mode de fixation a manqué d'équité, car la situation des producteurs les plus démunis (qui sont majoritaires) n'a pas été prise en compte. Ce sont des éléments que le gouvernement devra considérer s'il décidait de reprendre l'expérience du coton Bt.

\section{Références}

Baillard D. 2006. Le Burkina passe au coton transgénique. RFI, http:// www.rfi.fr/actufr/articles/083/article_47764.asp, consulté le 03/12/ 2006.

Coulibaly B, Niang M, Van der Pol F. 1993. Relations entre les pratiques d'amélioration des sols et les conditions socioéconomiques des exploitations agricoles au Mali. Club du Sahel, $52 \mathrm{p}$.

Barwale RB, Gadwal VR, Zehr U, Zehr B. 2004. Prospects for Bt cotton technology in India. AgBioForum 7: 23-26.

Berti F, Bioche F, Koama PE, Barry H, Ouédraogo DM. 2007. Étude sur l'analyse et les stratégies de développement durable de la filière coton. Rapport final. AGRER, Bruxelles. 267 p.

Bharathan G. 2000. Bt-cotton in India: anatomy of a controversy. Current Science 79: 1067-1075.

Cabanilla LS, Abdoulaye T, Sanders HJ. 2005. Economic cost of non-adoption of Bt cotton in West Africa: with special reference to Mali. International Journal of Biotechnology 7: 46-61.

CFDT. 1995. Filières cotonnières : nouvelles mesures fiscales depuis la dévaluation. Coton et Développement 13: 34-35.

Fok M. 1998. Cotton yield stagnation: addressing a common effect of various causes. In: Gillham FM, ed. World Cotton Research Conference 2. Athens, sept. 5-11, 1998: Petridis, P, Vol. 1, pp. 38-45.

FAO. 2004. Agricultural biotechnology. Meeting the needs of the poor? In: FAO, ed. The State of Food and Agriculture 2003-2004. Roma, pp. 1-39.

Fichet M. 1996. L'impact de la privatisation dans les pays africains de la zone Franc. Coton et Développement 20: 8-18.

Fok M. 2006. Conditions, résultats et perspectives d'utilisation du coton génétiquement modifié (coton Bt) dans les PED. Revue Tiers Monde: 773-798.

Fok M. 2007. Analyse multifactorielle de la baisse du rendement coton en zone CMDT : de la physiologie à l'idéologie. Montpellier, France: CIRAD.

Fok M. 2016. Impacts du coton-Bt sur les bilans financiers des sociétés cotonnières et des paysans au Burkina Faso. Cahiers Agricultures 25(3): 1-13.

Gakpo JO. 2018. Burkina Faso cotton industry wants to bring back GMO seeds. Available at: https://allianceforscience.cornell.edu/ blog/2018/02/burkina-faso-cotton-industry-wants-bring-backgmo-seeds/, consulté le 14/06/2019.

Glover D. 2010. Exploring the resilience of Bt Cotton's 'pro-poor success story'. Development and Change 41: 955-981.

Gouse M, Kirsten J, Shankar B, Thirtle C. 2005. Bt cotton in Kwazulu Natal: technological Triumph but institutional failure. Université de Pretoria.

Graff G, Roland-Holst D, Zilberman D. 2006. Agricultural biotechnology and poverty reduction in low-income countries. World Development 34: 1430-1445.

Gruère GP, Mehta-Bhatt P, Sengupta D. 2008. Bt cotton and farmer suicides in India. Reviewing the evidence. IFPRI, Washington, DC: $64 \mathrm{p}$.

Herring RJ. 2007. The Genomics revolution and development studies: science, poverty and politics. Journal of Development Studies 43: $1-30$.

Hofs JL, Fok M, Gouse M, Kirsten J. 2006. Diffusion du CGM dans une filière instable en Afrique du Sud et les leçons pour l'Afrique Zone Franc. Revue Tiers Monde: 799-823.

Morse S, Bennett R, Ismael Y. 2007. Isolating the 'farmer' effect as a component of the advantage of growing genetically modified 
varieties in developing countries: a Bt cotton case study from Jalgaon, India. The Journal of Agricultural Science 145: 491-500.

Morse S, Bennett R, Ismael Y. 2004. Why Bt cotton pays for smallscale producers in South Africa. e Biotechnology 22: 379-380.

Paarlberg RL. 2008. Starved for science: how biotechnology is being kept out of Africa. Harvard University Press.

Paarlberg RL. 2000. Governing the GM crop revolution: policy choices for developing countries. IFPRI: Food, Agriculture, and the Environment Discussion Paper, Washington DC, $44 \mathrm{p}$.

Pemsl DE, Waibel H, Orphal J. 2004. A methodology to assess the profitability of Bt-cotton: case study results from the state of Karnataka, India. Crop Protection 23: 1249-1257.

Persley GJ. 2006. Agricultural biotechnology and the poor: promethean science. Agricultural Biotechnology and the Poor. Washington DC: CGIAR, pp. 2-21.

Pray CE, Ma D, Huang J, Qiao F. 2001. Impact of Bt cotton in China. World Development 29: 813-825.

Qaim M, de Janvry A. 2005. Bt cotton and pesticide use in Argentina: economic and environmental effects. Environment and Development Economics 10: 179-200.
Qaim M, Subramanian A, Naik G, Zilberman D. 2006. Adoption of Bt cotton and impact variability: insights from India. Review of Agricultural Economics 28: 48-58.

Renaudin C. 2010. Vulnérabilité du paysannat cotonnier dans l'Est du Burkina Faso. Cahiers Agricultures 19(1): 54-59.

Renaudin C, Pelc H, Opois J. 2012. Cotonnier génétiquement modifié : l'expérience d'une campagne agricole au Burkina Faso. Cahiers Agricultures 21(6): 387-394.

Scoones I. 2006. Can biotechnology work for the poor? A sceptical look at the emerging 'consensus'. https://steps-centre.org/wpcontent/uploads/Agr_bio.pdf, consulté le 13/06/2014.

Smale M, Niane A, Zambrano P. 2010. Une revue des analyses de l'impact économique des plantes transgéniques dans les agricultures non industrialisées. Economie Rurale 315: 60-75.

Stone GD. 2004. Social constraints on crop biotechnology in developing countries. AgBioForum 7: 76-79.

Vitale JR, Glick H, Greenplate J, Abdennadher M, Traoré O. 2008. Second-generation Bt Cotton field trials in Burkina Faso: analyzing the potential benefits to West African farmers. Crop Science 48: 1958-1966.

Citation de l'article : Vognan G, Fok M. 2019. Performance différenciée du coton Bt en début de diffusion : cas du Burkina Faso. Cah. Agric. 28: 26 . 